\title{
Signatures of chiral symmetry restoration and its survival throughout the hadronic phase interactions
}

\author{
E.L. Bratkovskaya ${ }^{1,2, \star}$, P. Moreau ${ }^{1}$, A. Palmese ${ }^{3}$, W. Cassing ${ }^{3}$, E. Seifert ${ }^{3}$, and T. Steinert ${ }^{3}$ \\ ${ }^{1}$ Institute for Theoretical Physics, University of Frankfurt, Frankfurt, Germany \\ ${ }^{2}$ GSI Helmholtzzentrum für Schwerionenforschung GmbH, Darmstadt, Germany \\ ${ }^{3}$ Institute for Theoretical Physics, University of Giessen, Giessen, Germany
}

\begin{abstract}
The effect of the chiral symmetry restoration (CSR) on observables from heavy-ion collisions is studied in the energy range $\sqrt{s_{N N}}=3-20 \mathrm{GeV}$ within the PartonHadron-String Dynamics (PHSD) transport approach. The PHSD includes the deconfinement phase transition as well as essential aspects of CSR in the dense and hot hadronic medium, which are incorporated in the Schwinger mechanism for the hadronic particle production. We adopt different parametrizations of the nuclear equation of state from the non-linear $\sigma-\omega$ model, which enter in the computation of the quark scalar density for the CSR mechanism, in order to estimate the uncertainty in our calculations. For the pion-nucleon $\Sigma$-term we adopt $\Sigma_{\pi} \approx 45 \mathrm{MeV}$ which corresponds to a 'world average'. Our systematic studies show that chiral symmetry restoration plays a crucial role in the description of heavy-ion collisions at $\sqrt{s_{N N}}=3-20 \mathrm{GeV}$, realizing an increase of the hadronic particle production in the strangeness sector with respect to the non-strange one. We identify particle abundances and rapidity spectra to be suitable probes in order to extract information about CSR, while transverse mass spectra are less sensitive ones. Our results provide a microscopic explanation for the "horn" structure in the excitation function of the $K^{+} / \pi^{+}$ratio: the CSR in the hadronic phase produces the steep increase of this particle ratio up to $\sqrt{s_{N N}} \approx 7 \mathrm{GeV}$, while the drop at higher energies is associated to the appearance of a deconfined partonic medium.
\end{abstract}

\section{Introduction}

The strange particle production has always been suggested as one of the most sensitive observables in heavy-ion collisions (HIC) that could indicate the creation of a Quark-Gluon-Plasma (QGP) during the early stages of the reaction. The earliest suggested signature is the strangeness enhancement in $A+A$ collisions with respect to elementary $p+p$ collisions $[1,2]$. Later on, Gazdzicki and Gorenstein [3] proposed that a sharp rise and drop in the excitation function of the $K^{+} / \pi^{+}$ratio (so called "horn") should show up due to the appearance of a QGP phase at a center-of-mass energy $\sqrt{s_{N N}} \sim 7 \mathrm{GeV}$. Several statistical models [4-6] have succeeded in reproducing the trend of the experimental observation of the $\mathrm{K}^{+} / \pi^{+}$ratio and other strange to non-strange particle ratios, but they can provide only a statistical description of the heavy-ion collision process. On the other hand there was no conclusive interpretation of the "horn" from dynamical approaches for HIC, like microscopic transport models

\footnotetext{
^e-mail: E.Bratkovskaya@gsi.de
} 
[7, 8]. Only recently, the Parton-Hadron-String Dynamics (PHSD), a transport approach describing HIC on the basis of partonic, hadronic and string degrees-of-freedom, obtained a striking improvement on this issue when including chiral symmetry restoration (CSR) in the string decay for hadronic particle production [9-11].

Apart from deconfinement the chiral symmetry restoration addresses another aspect of the QCD phase diagram in the $\left(T, \mu_{B}\right)$-plane as an additional transition between a phase with broken and a phase with restored chiral symmetry. As in case of the QCD deconfinement phase transition, the boundaries of the CSR phase transition line are not well known. Lattice QCD (IQCD) calculations show that at vanishing baryon chemical potential $\left(\mu_{B}=0\right)$ the CSR takes place at roughly the same critical temperature and energy density as the deconfinement phase transition which is a crossover. At finite baryon chemical potential IQCD calculations cannot be performed due to the sign problem and one must rely on effective models (or extrapolations) in order to study the QCD phase transitions [12-14]. Different models support the idea that at finite chemical potential a partially restored phase is achieved before the deconfinement occurs [15]. In order to distinguish the two phases of such a transition, effective models use the scalar quark condensate $\langle\bar{q} q\rangle$ as an order parameter. As the baryon density and temperature increase, the scalar quark condensate $\langle\bar{q} q\rangle$ is expected to decrease from a nonvanishing value in the vacuum to $\langle\bar{q} q\rangle \approx 0$ which corresponds to CSR. Since $\langle\bar{q} q\rangle$ is not a measurable quantity, it is crucial to determine experimental observables which are sensitive to this quantity. The dilepton spectroscopy has been in the focus in this respect since in a chirally restored phase the spectral functions of the the $\rho$ - and the $a_{1}$-meson should become identical. However, no clear evidence has been achieved so far [16]. On the other hand, the strangeness production at Alternating-Gradient Synchrotron (AGS) and lower SPS energies was suggested to be a signature of CSR [9].

In this contribution we will report on the main results from Refs. [9-11] where a systematic study has been performed on effects of the CSR on final particle distributions within the PHSD approach.

\section{Reminder of the PHSD transport approach}

The Parton-Hadron-String Dynamics (PHSD) is a microscopic covariant dynamical approach for strongly interacting systems in and out-of equilibrium [17-20] incorporating also the self-generated retarded electromagnetic fields [21]. It is a transport approach which goes beyond the quasi-particle approximation, since it is based on Kadanoff-Baym equations for the Green 's functions in phase-space representation in first-order gradient expansion [22, 23]. Including both hadronic and partonic phase as well as a transition between the effective degrees-of-freedom, PHSD is capable to describe the full time evolution of a relativistic heavy-ion collision. The theoretical description of the partonic degreesof-freedom (quarks and gluons) is realized in line with the Dynamical-Quasi-Particle Model (DQPM) [23] which reproduces 1 QCD results in thermodynamical equilibrium and provides the properties of the partons, i.e. masses and widths in their spectral functions. In equilibrium the PHSD reproduces the partonic transport coefficients such as shear and bulk viscosities or the electric conductivity from 1QCD calculations as well. For a review on PHSD we refer the reader to Ref. [24]. We further note that the pure hadronic phase in PHSD is equivalent to the Hadron-Strings Dynamics (HSD) approach [25]. Accordingly, the comparison between PHSD and HSD calculations allows us to disentangle the role of the QGP phase in heavy-ion collisions.

The PHSD approach has been tested for different colliding systems $(\mathrm{p}+\mathrm{p}, \mathrm{p}+\mathrm{A}, \mathrm{A}+\mathrm{A})$ in a wide range of bombarding energy, from AGS to LHC energies, and has been able to describe a large number of experimental observables, such as charged particle spectra, collective flow coefficients $v_{n}$ as well as electromagnetic probes such as photons and dileptons [24]. More recently, it has been also shown to provide a microscopic description of the maximum in the $K^{+} / \pi^{+}$ratio in central nucleus-nucleus collisions [9-11]. 


\subsection{String fragmentation in PHSD}

The string formation and decay represents the dominant particle production mechanism in nucleusnucleus collisions for bombarding energies from $2 \mathrm{AGeV}$ to $160 \mathrm{AGeV}$. In PHSD, the primary hard scatterings between nucleons are described by string formation and decay in the FRITIOF Lund model [26]. A string is an excited color-singlet state, which is composed of two string ends corresponding to the leading constituent quarks of the colliding hadrons and a color flux tube in between. As the string ends recede, virtual $q \bar{q}$ or $q q \bar{q} \bar{q}$ pairs are produced in the uniform color field, causing the breaking of the string. Finally, the string decays into mesons or baryon-antibaryon pairs with formation time $\tau_{f} \sim 0.8 \mathrm{fm} / \mathrm{c}$ (in the rest-frame of the string).

In the string decay, the flavor of the produced quarks is determined via the Schwinger formula [27], which defines the production probability of massive $s \bar{s}$ pairs with respect to light-flavor production of $(u \bar{u}, d \bar{d})$ pairs:

$$
\frac{P(s \bar{s})}{P(u \bar{u})}=\frac{P(s \bar{s})}{P(d \bar{d})}=\gamma_{s}=\exp \left(-\pi \frac{m_{s}^{2}-m_{u, d}^{2}}{2 \kappa}\right),
$$

with $\kappa \approx 0.176 \mathrm{GeV}^{2}$ representing the string tension and $m_{u, d, s}$ denoting the constituent quark masses for strange and light quarks. For the constituent quark masses $m_{u} \approx 0.35 \mathrm{GeV}$ and $m_{s} \approx 0.5 \mathrm{GeV}$ in the vacuum, the production of strange quarks is suppressed by a factor of $\gamma_{s} \approx 1 / 3$ with respect to the light quarks, which is the default setting in the FRITIOF routines.

\subsection{Modeling of the chiral symmetry restoration}

In Refs. [9, 10] the PHSD has been extended to include CSR in the string decay in a hadronic environment of finite baryon and meson density. Here we recall the main aspect of this extension which is based on the Hellman-Feynman theorem for the scalar quark condensate [28]. Accordingly, a linear decrease of the scalar quark condensate $\langle\bar{q} q\rangle$ - which is nonvanishing in the vacuum due to a spontaneous breaking of chiral symmetry - is expected with baryon density $\rho_{B}$ towards a chiral symmetric phase characterized by $\langle\bar{q} q\rangle \approx 0[29,30]$. This decrease of the scalar quark condensate is expected also to lead to a change of the hadron properties with density and temperature, i.e. in a chirally restored phase the vector and axial vector currents should become equal [31]. Since the scalar quark condensate $\langle\bar{q} q\rangle$ is not a direct observable, its manifestations should also be found indirectly in different hadronic abundances and spectra or particle ratios like $K^{+} / \pi^{+},\left(\Lambda+\Sigma^{0}\right) / \pi^{-}$etc. as advocated in Ref. [9].

In leading order the scalar quark condensate $\langle\bar{q} q\rangle$ can be evaluated in a dynamical calculation as follows [32]:

$$
\frac{\langle\bar{q} q\rangle}{\langle\bar{q} q\rangle_{V}}=1-\frac{\Sigma_{\pi}}{f_{\pi}^{2} m_{\pi}^{2}} \rho_{S}-\sum_{h} \frac{\sigma_{h} \rho_{S}^{h}}{f_{\pi}^{2} m_{\pi}^{2}},
$$

where $\sigma_{h}$ stands for the $\sigma$-commutator of the relevant mesons $h,\langle\bar{q} q\rangle_{V}$ represents the vacuum condensate, $\Sigma_{\pi} \approx 45 \mathrm{MeV}$ is the pion-nucleon $\Sigma$-term and $f_{\pi}$ and $m_{\pi}$ are the pion decay constant and pion mass, respectively.

In Eq. (2), the quantities $\rho_{S}$ and $\rho_{S}^{h}$ denote the nucleon scalar density and the scalar density for a meson of type $h$, respectively. The scalar density of mesons $h$ is evaluated in the independent-particle approximation as:

$$
\rho_{S}^{h}(x)=\frac{(2 s+1)(2 \tau+1)}{(2 \pi)^{3}} \int \mathrm{d}^{3} p \frac{m_{h}}{\sqrt{\mathbf{p}^{2}+m_{h}^{2}}} f_{h}(x, \mathbf{p}),
$$


where $f_{h}(x, \mathbf{p})$ denotes the meson phase-space distribution $(x=(\mathbf{r}, t))$ and $s, \tau$ refer to the discrete spin and isospin quantum numbers, respectively. Moreover, the vacuum scalar condensate $\langle\bar{q} q\rangle_{V}=$ $\langle\bar{u} u\rangle_{V}+\langle\bar{d} d\rangle_{V} \approx 2\langle\bar{u} u\rangle_{V}$ can be computed according to the Gell-Mann-Oakes-Renner (GOR) relation $[33,34]$,

$$
f_{\pi}^{2} m_{\pi}^{2}=-\frac{1}{2}\left(m_{u}^{0}+m_{d}^{0}\right)\langle\bar{q} q\rangle_{V},
$$

and gives $\langle\bar{q} q\rangle_{V} \approx-3.2 \mathrm{fm}^{-3}$ for the bare quark masses $m_{u}^{0}=m_{d}^{0} \approx 7 \mathrm{MeV}$. Finally, in Eq. (2) the nucleon scalar density $\rho_{S}$ has to be determined in a suitable model with interacting degrees-offreedom in order to match our knowledge on the nuclear EoS at low temperature and finite density. A proper (and widely used) approach is the non-linear $\sigma-\omega$ model for nuclear matter where $\rho_{S}$ is defined as:

$$
\rho_{S}(x)=\frac{d}{(2 \pi)^{3}} \int \mathrm{d}^{3} p \frac{m_{N}^{*}}{\sqrt{\mathbf{p}_{N}^{* 2}+m_{N}^{* 2}}} f_{N}(x, \mathbf{p}),
$$

where $m_{N}^{*}$ and $p_{N}^{*}$ denote the effective mass and four-momentum, respectively, and $f_{N}(x, \mathbf{p})$ the phasespace occupation of a nucleon while the degeneracy factor is $d=4$. In fact, in the non-linear $\sigma-\omega$ model the nucleon mass is modified due to the scalar interaction with the medium:

$$
m_{N}^{*}(x)=m_{N}^{v}-g_{s} \sigma(x),
$$

where $m_{N}^{v}$ denotes the nucleon mass in vacuum and $\sigma(x)$ is the scalar field which mediates the interaction between the nucleons and the medium with the coupling $g_{s}$. In order to calculate $\rho_{S}$, we need to determine the value of the scalar field $\sigma(x)$ at each space-time point $x$. This is done via the non-linear gap equation $[35,36]$ :

$$
m_{\sigma}^{2} \sigma(x)+B \sigma^{2}(x)+C \sigma^{3}(x)=g_{s} \rho_{S}(x)=g_{s} d \int \frac{\mathrm{d}^{3} p}{(2 \pi)^{3}} \frac{m_{N}^{*}(x)}{\sqrt{\mathbf{p}^{2}+m_{N}^{* 2}}} f_{N}(x, \mathbf{p}),
$$

since for matter at rest we have $\mathbf{p}^{*}=\mathbf{p}$. In Eq. (7) the self-interaction of the $\sigma$-field is included up to the forth order. The parameters $g_{s}, m_{\sigma}, B, C$ are fixed in order to reproduce the values of the nuclear matter quantities at saturation, i.e. the saturation density, the binding energy per nucleon, the compression modulus, and the effective nucleon mass. Actually, there are different sets for these quantities (NL1,NL2,NL3) that lead to slightly different saturation properties. For details we refer the reader to Ref. [10].

The main idea in Refs. [9, 10] is to consider effective masses for the dressed quarks in the Schwinger formula (1) for the string decay in a hot and dense medium. The effective quark masses can be expressed in terms of a scalar coupling to the quark condensate $\langle\bar{q} q\rangle$ in first order as follows:

$$
m_{s}^{*}=m_{s}^{0}+\left(m_{s}^{v}-m_{s}^{0}\right) \frac{\langle\bar{q} q\rangle}{\langle\bar{q} q\rangle_{V}}, \quad m_{q}^{*}=m_{q}^{0}+\left(m_{q}^{v}-m_{q}^{0}\right) \frac{\langle\bar{q} q\rangle}{\langle\bar{q} q\rangle_{V}},
$$

with $m_{s}^{0} \approx 100 \mathrm{MeV}$ and $m_{q}^{0} \approx 7 \mathrm{MeV}$ for the bare quark masses. In Eq. (8) the effective masses decrease from the vacuum values with decreasing scalar condensate $\langle\bar{q} q\rangle$ to the constituent masses. This adaptation of the Schwinger formula in case of a hot and dense medium implies a modification of the flavor production factors in Eq. (1). In an actual nucleus-nucleus collision, PHSD incorporates a dynamical calculation of all these features for each cell in space-time:

- the scalar density $\rho_{S}$ is determined by solving the gap equation (7) for the $\sigma$-field;

- the scalar condensate $\langle\bar{q} q\rangle$ is then computed via Eq. (2); 
- the effective masses $m_{q}^{*}, m_{s}^{*}$ are calculated according to Eqs. (8) and plugged in the Schwinger formula (1) in order to compute the flavor production ratios for the string decay.

We stress that, once the nucleon scalar density $\rho_{S}$ and $\Sigma_{\pi}(\approx 45 \mathrm{MeV})$ are fixed, there is no additional 'parameter' in the PHSD3.3 compared to the previous version PHSD3.2 that has been employed for a couple years for the analysis of relativistic heavy-ion reactions [24]. We note that the sets NL1 and NL3 have the same compression modulus $K$ but differ in the effective mass $m^{*} / m$ at saturation density whereas NL1 and NL2 have the same effective mass but differ in the compression modulus $K$. By comparing the results from NL1, NL2 and NL3 we are able to explore separately the effects from the effective mass and compression modulus. For a detailed discussion of the flavor ratio $\gamma_{s}$ versus energy density for the different parameter sets NL1, NL2 and NL3 we refer the reader to Ref. [10].

\section{Application to nucleus-nucleus collisions}

In this section we present results for the most abundant particles from HIC at AGS and SPS energies for different nuclear equations of state in order to estimate the uncertainties of our approach. We briefly recall the main results on the hadron rapidity spectra discussed in detail in Ref. [10]. In general, the restoration of chiral symmetry gives an enhancement of the strange particle yields both for mesons and baryons at AGS and lower SPS energies. On the other hand, it produces a slight decrease in the number of pions at midrapidity due to the suppression of pions in the string decays in favor of strange hadrons. The proton rapidity spectra do not show any sensible variation, in fact the CSR as implemented in PHSD modifies essentially the chemistry of the newly produced particles in the string decay and has a minor impact the dynamics of the nucleons, which in the string picture are associated to the string ends of the primary interactions in the system. The inclusion of the CSR is essential in order to correctly reproduce the strange particle rapidity spectra especially for $\left(\Lambda+\Sigma^{0}\right)$ hyperons and $K^{+}$mesons. We note that our calculations for the hadron rapidity spectra are in good agreement with experimental observation [10].

The results from PHSD with CSR using three different parametrizations for the nuclear equation of state, i.e. NL3, NL2 and NL1 show that the general features of the strangeness enhancement hold for all parametrizations; in particular the NL1 set provides larger values for the strange particle rapidity spectra at midrapidity since the scalar nucleon density is larger in this case. The difference between the parametrizations represents the uncertainty of our results related to CSR as implemented in PHSD (see below). At the top SPS energy $E_{L a b}=158 \mathrm{AGeV}$ the CSR does not play a significant role, since the dynamics is dominated by the QGP phase. Thus, there is no appreciable difference between the results with and without CSR for the different nuclear EoS. Our results for $K^{+}$are slightly lower with respect to the experimental data [10], however, the $\left(\Lambda+\Sigma^{0}\right), \pi^{-}$and $K^{-}$as well as the protons are correctly reproduced.

We, furthermore, recall that in earlier HSD calculations (without a partonic phase) the slopes of the transverse mass distributions of kaons and antikaons have been severely underestimated [8]. This improves drastically when a partonic phase is incorporated as in PHSD. However, the effect from CSR on the transverse mass spectra is only very moderate since the CSR acts directly on the chemistry and not so much on the dynamics of the Schwinger mechanism [10].

We now summarize our findings with respact to the excitation function of the particle ratios $K^{+} / \pi^{+}$, $K^{-} / \pi^{-}$and $\left(\Lambda+\Sigma^{0}\right) / \pi$ at midrapidity from $5 \%$ central $\mathrm{Au}+\mathrm{Au}$ collisions. In Fig. 1 (1.h.s.) we show the calculations for the following three scenarios: the default PHSD without CSR (blue dotted line), PHSD including CSR with NL3 and NL1 as parameter sets for the nuclear EoS from the non-linear $\sigma-\omega$ model (red solid and green dashed lines, respectively). The shaded area displays the uncertainties of our calculations from the two scenarios for the nuclear EoS since the results from the parameter set NL2 are always in between those from NL1 and NL3 [10]. 

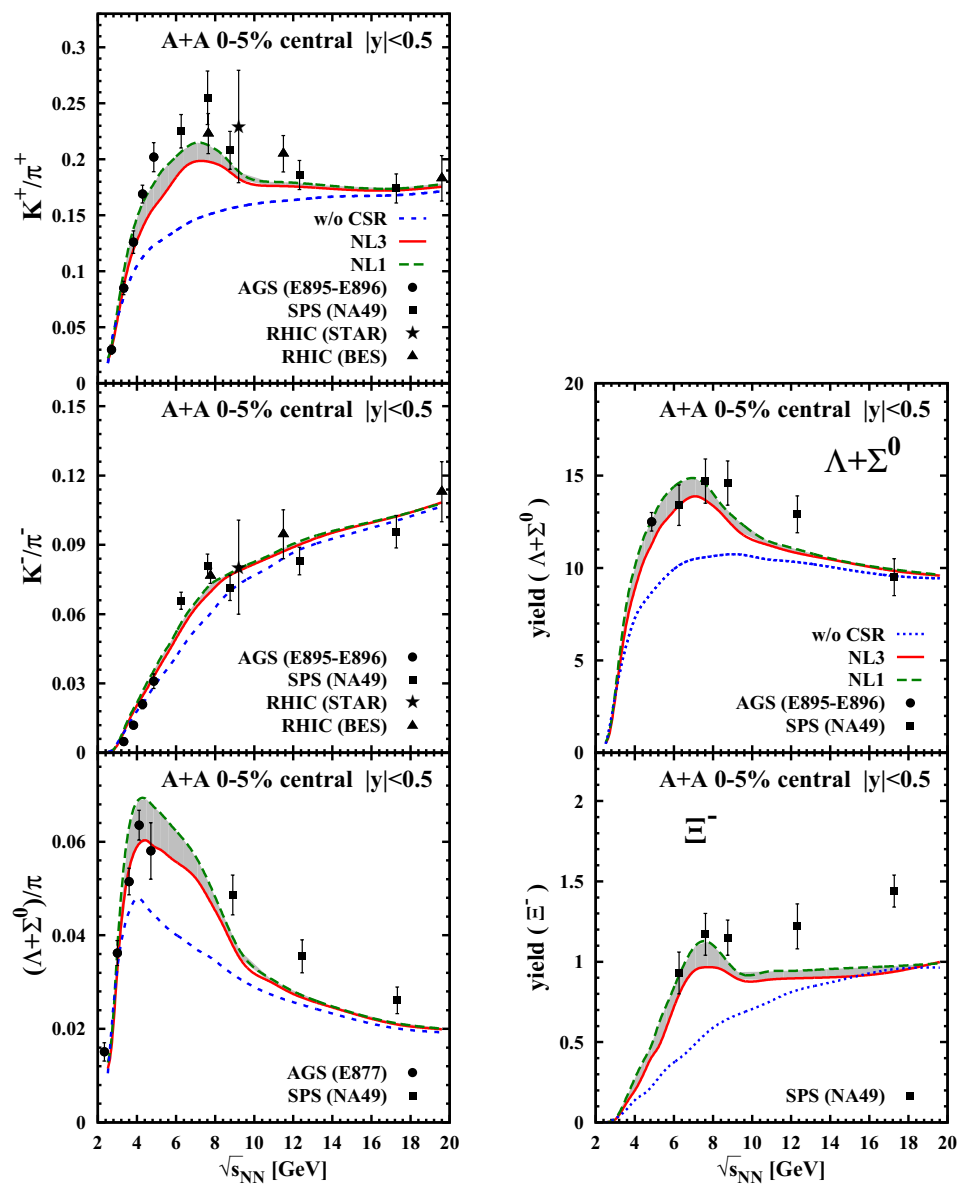

Figure 1. (1.h.s.) The ratios $K^{+} / \pi^{+}, K^{-} / \pi^{-}$and $\left(\Lambda+\Sigma^{0}\right) / \pi$ at midrapidity from $5 \%$ central Au+Au collisions as a function of the invariant energy $\sqrt{s_{N N}}$ up to the top SPS energy in comparison to the experimental data from [3840]. The grey shaded area represents the results from PHSD including CSR taking into account the uncertainty from the parameters of the $\sigma-\omega$-model for the EoS. (r.h.s.) The yields of $\left(\Lambda+\Sigma^{0}\right)$ and $\Xi^{-}$at midrapidity from $5 \%$ central $\mathrm{Au}+\mathrm{Au}$ collisions as a function of the invariant energy $\sqrt{s_{N N}}$ up to the top SPS energy in comparison to the experimental data from Refs. [37, 40].

As described in Refs. [9, 10], the inclusion of CSR in PHSD is responsible for the strong strangeness enhancement at AGS and low SPS energies. The experimental observations of the ratios $K^{+} / \pi^{+}$and $\left(\Lambda+\Sigma^{0}\right) / \pi$ show the well-known "horn" structure, which is reproduced by the PHSD calculations with CSR. In fact, CSR gives rise to a steep increase of these ratios at energies lower than $\sqrt{s_{N N}} \approx 7 \mathrm{GeV}$, while the drop at larger energies is associated to the appearance of a deconfined partonic medium. The NL1 parameter set produces a sharper peak both in the $K^{+} / \pi^{+}$and in the $\left(\Lambda+\Sigma^{0}\right) / \pi$ excitation functions with $\mathrm{a} \approx 10 \%$ maximum increase with respect to the NL3 result. We point out 
that even adopting different parametrizations for the $\sigma-\omega$ model, we recover the same "horn" feature. This supports the reliability of the CSR mechanism as implemented in the PHSD model.

At AGS energies, the energy dependencies of the ratios $K^{+} / \pi^{+}$and $\left(\Lambda+\Sigma^{0}\right) / \pi$ are closely connected, since $K^{+}$and $\Lambda$ (or $\Sigma^{0}$ ) are mostly produced in pairs due to strangeness conservation. On the other hand, the excitation function of the $K^{-} / \pi^{-}$ratio does not show any peak, but smoothly increases as a function of $\sqrt{s_{N N}}$. In fact, especially at AGS energies, the antikaon production differs substantially from the production of $K^{+}$and $\Lambda$, which occurs predominantly via string formation. The antikaons are produced dominantly via secondary meson-baryon interactions by flavor exchange and their production is suppressed with respect to the $\Lambda$ hyperons that carry most of the strange quarks. This is the reason why the inclusion of chiral symmetry restoration provides a substantial enhancement of the $K^{+} / \pi^{+}$and $\left(\Lambda+\Sigma^{0}\right) / \pi$ excitation functions and a smaller change on the $K^{-} / \pi^{-}$ratio. We also notice that there is no sizeable difference between the NL1 and NL3 results for the $K^{-} / \pi^{-}$ ratio. At top SPS energies the strangeness is produced predominantly by the hadronization of partonic degrees-of-freedom, thus our results for all the ratios do not show an appreciable sensitivity to the nuclear EoS and the calculations with and without CSR tend to merge at $\sqrt{s_{N N}} \approx 20 \mathrm{GeV}$.

Finally, in Fig. 1 (r.h.s.) we present the yields of $\left(\Lambda+\Sigma^{0}\right)$ and $\Xi^{-}$at midrapidity from $5 \%$ central $\mathrm{Au}+\mathrm{Au}$ collisions as a function of the invariant energy $\sqrt{s_{N N}}$ in comparison to the available data from Refs. [37, 40]. We recover a "horn" structure, similar to that shown in Fig. 1 (1.h.s.) for the energy dependence of the strange to non-strange particle ratios. A sensitivity on the nuclear model parametrizations persists at low energy, while in the top SPS energy regime the results corresponding to the different scenarios merge. The comparison with the available data at $\sqrt{s_{N N}}<8 \mathrm{GeV}$ supports the validity of the CSR picture, while at larger energies we under-estimate the experimental observations for $\Xi^{-}$baryons. We mention that this discrepancy is not due to the CSR mechanism, since it does not play an essential role in the high-energy regime.

\section{Summary}

We point out that our PHSD calculations provide a microscopic interpretation of the "horn" structure in the excitation function of the $K^{+} / \pi^{+}$ratio in central $\mathrm{Au}+\mathrm{Au}$ (or $\mathrm{Pb}+\mathrm{Pb}$ ) collisions. The steep rise of this ratio at AGS energies is associated to chiral symmetry restoration (CSR) in the hadronic phase, while the drop at higher SPS energies is due to the appearance of the QGP phase in an increasing volume of the interaction region. We have found an analogous energy dependence for the $\left(\Lambda+\Sigma^{0}\right) / \pi$ ratio, while the excitation function of the $K^{-} / \pi^{-}$ratio does not show any explicit peak. In general, the PHSD results obtained with the inclusion of CSR are in a good agreement with the available data for all observables analyzed, while calculations without CSR fail substantially.

The microscopic PHSD studies support the idea that CSR occurs in hadronic systems with high temperatures and densities before the deconfinement phase transition takes over. We suggest that the strange particle spectra and yields are suitable signatures to study the properties of CSR in HICs in future also as a function of system size and centrality as advocated in Ref. [10].

\section{Acknowledgments}

The authors acknowledge inspiring discussions with J. Cleymans, M. Gazdzicki, M. Gorenstein and O. Linnyk and thank the Helmholtz International Center for FAIR (HIC for FAIR), the Helmholtz Graduate School for Hadron and Ion Research (HGS-HIRe), the Helmholtz Research School for Quark Matter Studies in Heavy-Ion Collisions (H-QM), and the Bundesministerium für Bildung und Forschung (BMBF) for support. The computational resources have been provided by the LOEWE-CSC. 


\section{References}

[1] J. Rafelski and B. Müller, Phys. Rev. Lett. 48, 106 (1982).

[2] R. Stock, J. Phys. G 28, 1517 (2002).

[3] M. Gazdzicki and M.I. Gorenstein, Acta Phys. Polon. B 30, 2705 (1999).

[4] J. Cleymans et al., Phys. Lett. B 615, 50 (2005).

[5] A. Andronic, P. Braun-Munzinger, and J. Stachel, Phys. Lett. B 673, 142 (2009).

[6] K. A. Bugaev et al., Europhys. Lett. 104, 22002 (2013).

[7] J. Geiss, W. Cassing, and C. Greiner, Nucl. Phys. A 644, 107 (1998).

[8] E. L. Bratkovskaya et al., Phys. Rev. C 69, 054907 (2004); Phys. Rev. Lett. 92, 032302 (2004).

[9] W. Cassing, A. Palmese, P. Moreau, and E.L. Bratkovskaya, Phys. Rev. C 93, 014902 (2016).

[10] A. Palmese et al., Phys. Rev. C 94, 044912 (2016).

[11] E.L. Bratkovskaya, W. Cassing, P. Moreau, and A. Palmese, Eur. Phys. J. A 52, 219 (2016).

[12] P. Senger et al., Lect. Notes Phys. 814, 681 (2011).

[13] C. S. Fischer, L. Fister, J. Luecker, J. M. Pawlowski, Phys. Lett. B 732, 273 (2014).

[14] T. K. Herbst, J. M. Pawlowski, and B. J. Schaefer, Phys. Rev. D 88, 014007 (2013).

[15] L. McLerran and R. D. Pisarski, Nucl. Phys. A 796, 83 (2007).

[16] R. Rapp, Adv. High Energy Phys. 2013, 148253 (2013).

[17] W. Cassing and E. L. Bratkovskaya, Phys. Rev. C 78, 034919 (2008).

[18] W. Cassing and E. L. Bratkovskaya, Nucl. Phys. A 831, 215 (2009).

[19] E. L. Bratkovskaya et al., Nucl. Phys. A 856, 162 (2011).

[20] V. P. Konchakovski et al., Phys. Rev. C 85, 044922 (2012); Phys. Rev. C 85, 011902 (2012).

[21] V. D. Toneev et al., Phys. Rev. C 95, 034911 (2017).

[22] L. P. Kadanoff and G. Baym, Quantum Statistical Mechanics, Benjamin, New York, 1962.

[23] W. Cassing, Eur. Phys. J. ST 168, 3 (2009); Nucl. Phys. A 795, 70 (2007).

[24] O. Linnyk, E. Bratkovskaya, and W. Cassing, Prog. Part. Nucl. Phys 87, 50 (2016).

[25] W. Cassing and E. L. Bratkovskaya, Phys. Rep. 308, 65 (1999).

[26] B. Andersson, G. Gustafson, and H. Pi, Z. Phys. C 57, 485 (1993).

[27] J. Schwinger, Phys. Rev. 83, 664 (1951).

[28] T. D. Cohen, R. J. Furnstahl, and D. K. Griegel, Phys. Rev. C 45, 1881 (1992).

[29] U. Vogl and W. Weise, Prog. Part. Nucl. Phys. 27, 195 (1991).

[30] M. C. Birse, J. Phys. G 20, 1537 (1994).

[31] V. Koch, Int. J. Mod. Phys. E 6, 203 (1997).

[32] B. Friman, W. Nörenberg, and V. D. Toneev, Eur. Phys. J. A 3, 165 (1998).

[33] M. Bando, T. Kugo, and K. Yamawaki, Phys. Rep. 164, 217 (1988).

[34] T. D. Cohen, R. J. Furnstahl, D. K. Griegel, and X. Jin, Prog. Part. Nucl. Phys. 35, 221 (1995).

[35] J. Boguta and A. R. Bodmer, Nucl. Phys. A 292, 413 (1977).

[36] A. Lang et al., Z. Phys. A 340, 287 (1991).

[37] S. Albergo et al., Phys. Rev. Lett. 88, 062301 (2002).

[38] J. L. Klay et al., Phys. Rev. C 68, 054905 (2003).

[39] B. I. Abelev et al., Phys. Rev. C 81, 024911 (2010); M. M. Aggarwal et al., Phys. Rev. C 83, 024901 (2011).

[40] C. Alt et al., Phys. Rev. C 78, 034918 (2008). 